\title{
A CONTRIBUTION RELATIVE TO THE STRUCTURE OF COLLAGEN
}

By John Beek, jr.

ABSTRACT

The quantity of gaseous hydrogen chloride reacting with collagen was measured, under various conditions by three methods. The seven combining weights thus obtained, together with two similar values for gelatin, are used to find the number of nitrogen atoms in the unit of structure of the collagen. This number is found to be 38 , or possibly a multiple of 38 .

\section{CONTENTS}

I. Introduction

II. Methods and results

III. Discussion of results

552

IV. Conclusions.

\section{INTRODUCTION}

A study of the fundamental principles governing tanning processes requires a more definite knowledge of the structure of collagen, the chief constituent of hides.

Collagen is generally considered to be a chemical species in the classical sense, but the evidence on this point is not conclusive. The work of Thomas ${ }^{1}$ and Kelly on the reactions of collagen with chromium and iron salts shows that it is probable that collagen is a definite substance.

This research was undertaken in connection with the problem of determining the combining weight of collagen with acids in dilute aqueous solution. It is believed that a knowledge of the number of nitrogen atoms occurring in a unit of the collagen structure would facilitate the accurate determination of this combining weight, as it is the nitrogen-containing groups which react basically in collagen. If a definite integral value is obtained for this number it is simultaneously demonstrated that collagen is a chemical species. Thus it is unnecessary to prove independently that collagen is a definite substance if a positive result is obtained in a research on one of its structural characteristics.

If the combining ratios in a series of compounds formed from collagen could be shown to bear a stoichiometrical relation to the nitrogen content of the collagen, some factor of the number of nitrogen atoms in a structural unit of the collagen would be known.

In the present work the quantity of $\mathrm{HCl}$ gas which reacts with collagen is determined under various conditions. It is demonstrated that a whole-number relation exists among the various combining ratios which are obtained. As Bancroft ${ }^{2}$ has shown that gaseous

1 Ind. Eng. Chem., vol 14, p. 621, 1922; and Ind Eng. Chem. vol, 20, p. 632, 1928.

2 J. Phys. Chem., vol. 34, pp. 449 and 753, 1930. 
$\mathrm{HCl}$ reacts only with the nitrogen- containing groups in polypeptides, and that not more than $1 \mathrm{~mol}$ of $\mathrm{HCl}$ per nitrogen atom reacts, it is possible to calculate from the above series of results the number of nitrogen atoms in a unit of the collagen structure.

\section{METHODS AND RESULTS}

The collagen used in this work was prepared in this laboratory. Steer hide was treated as suggested by Dr. J. A. Wilson, ${ }^{3}$ except that instead of shredding the material after splitting it was cut into small pieces and deashed by putting it in water and adding hydrochloric acid until the solution remained acid to methyl red. The collagen was then washed thoroughly. Ammonia was then added until the solution remained basic to methyl red, after which the washing was repeated. This process was continued until the collagen had been in acid solution three times and in basic solution three times. The resulting collagen contained 17.8 per cent $\mathrm{N}, 0.02$ per cent ash, and 0.02 per cent $\mathrm{Cl}$.

The results of the determinations are expressed in terms of the fraction of the total nitrogen which reacted with $\mathrm{HCl}$. The letter $f$ will be used to denote this fraction. The values of $f$ are calculated by taking the ratio of the number of gram atomic weights of chlorine, in the sample after the reaction, to the number of gram atomic weights of nitrogen in the sample.

A description of the methods used in making the determinations follows:

1. A sample of collagen was dried at $100^{\circ} \mathrm{C}$. for 2 days, and then allowed to remain in an atmosphere of hydrogen chloride for 151 days at room temperature $\left(15^{\circ}\right.$ to $35^{\circ}$ C. $)$. At the end of this time the hydrogen chloride was swept with a stream of air out of the vessel containing the collagen, the collagen salt was dissolved in cold water, and the chloride was titrated with $\mathrm{AgNO}_{3}$ solution, using $\left(\mathrm{NH}_{4}\right)_{3} \mathrm{AsO}_{4}$ as an indicator. The value of $f$ was 0.395 .

2. A sample of collagen was dried over $\mathrm{P}_{2} \mathrm{O}_{5}$ for 2 months and then put in an atmosphere of $\mathrm{HCl}$ for 45 days. The total chloride in the sample was then determined as in method 1. The value of $f$ was 0.398 .

3. A sample of collagen dried over $\mathrm{P}_{2} \mathrm{O}_{5}$ for 44 days was kept in an atmosphere of $\mathrm{HCl}$ for 3 days. At the end of this period the rate of combination of the $\mathrm{HCl}$ with the collagen was so low as not to be easily measurable by the volume change. The sample was transferred to a vessel containing sodium in an atmosphere of hydrogen. The sample was suspended on a glass rod passing through the top of the vessel, so that it could be weighed without making a large opening. The pieces of sodium were cut at intervals of about 10 days in order to expose fresh surfaces to the atmosphere in the vessel. After 49 days, the rate of loss of weight of the collagen sample being less than $0.1 \mathrm{mg}$ per day, the sample was dissolved in water and the chloride titrated with $\mathrm{AgNO}_{3}$, as in method 1, above. This gave 0.107 as the value of $f$.

4. The sodium from the vessel used in experiment 3 was put with the adhering salts, into an atmosphere of moist air and left until no metallic sodium remained. The resulting sodium salts were dissolved in water, and the chloride titrated as in methods 1 and 2 above. The quantity given by this titration was added to that of experiment 3 ,

3 The Chemistry of Leather Manufacture, vol. 1, p. 90. 
giving the total chloride in the collagen sample when it was put in the vessel with the sodium. The resulting value of $f$ was 0.317 .

5. This method is similar to that employed by Dr. W. D. Bancroft ${ }^{4}$ in measuring the quantity of $\mathrm{HCl}$ reacting with various proteins over a range of pressure. It consists essentially in admitting successive quantities of $\mathrm{HCl}$ into an evacuated vessel containing the protein, and measuring the equilibrium pressure in the vessel after each addition. These measurements permit the construction of a phase diagram for the 2-component system over the range of pressure studied. Not enough data were obtained to make possible the construction of a complete phase diagram for the samples used, but definite regions in which the quantity of $\mathrm{HCl}$ combined did not vary with the pressure were established at values of $f$, of 0.184 , and 0.236 . The corresponding pressures were in the region of 12 and $30 \mathrm{~mm}$, respectively. The value 0.236 was obtained in two separate determinations.

6. A sample of leather tanned with chestnut wood extract was used in this determination. The grain and flesh surfaces were split off, after which the material was washed in a shaking machine with about 30 changes of distilled water, and then in a water reflux apparatus for a month. The leather contained 9.82 per cent nitrogen. A dropping funnel of $60 \mathrm{ml}$ capacity with the stem cut off about $5 \mathrm{~cm}$ from the stopcock, was used as a container for the sample, which weighed about $4.3 \mathrm{~g}$. The bulb, containing the sample (dried at $100^{\circ} \mathrm{C}$.) was filled with $\mathrm{HCl}$. After 12 days the pressure was reduced to $15 \mathrm{~mm}$, and repeatedly brought to that value, as the leather gave off $\mathrm{HCl}$, until the pressure remained constant at that point. The container was then weighed, with the gas at $15 \mathrm{~mm}$ pressure and the leather in it. The process was then repeated. The two weights checked to within $3 \mathrm{mg}$, giving a value of 0.343 for $f$, after correction for the $\mathrm{HCl}$ taken up by the tannin.

7. Deashed gelatin was used for this determination. The sample was used without drying, containing 13.7 per cent moisture. It was put in an atmosphere of $\mathrm{HCl}$ for seven days, after which the total chloride was determined as in method 1 . The result was $f=0.633$.

8. This is the same as method 7 except that the sample was heated at $180^{\circ} \mathrm{C}$. for two days before being put in $\mathrm{HCl}$. The result was $f=0.105$.

The work on gelatin is included because of the close relation between collagen and gelatin.

Table 1 gives the experimental results.

TABLE 1.-Showing the fraction of the total $\mathrm{N}$ reacting with $\mathrm{HCl}$

\begin{tabular}{|c|c|}
\hline No. & $f$ \\
\hline 1 & 0.395 \\
2 & .398 \\
3 & .107 \\
4 & .317 \\
$5-(1)$ & .184 \\
$5-(2)$ & .236 \\
$5-(3)$ & .236 \\
6 & .343 \\
7 & .633 \\
8 & .105 \\
\hline
\end{tabular}




\section{DISCUSSION OF RESULTS}

The whole number relation among the results may be shown by dividing all the values of $f$ by the smallest value. For cases in which the values apparently occur in pairs, the mean of the two is taken, and later used with a weight of two. The resulting quotients fall approximately on quarters of integers. Thus, if they are multiplied by four they are approximately integers, which may be denoted as $a_{1}, a_{2}$, etc. Then all of the ratios $f / a$ are about equal, and represent the smallest fraction of the nitrogen which was found to react. The weighted mean of the values of this smallest fraction will be called $f_{0}$. The numerical values of the quantities which have been discussed are presented in Table 2.

The deviation of the values of $f / f_{0}$ from the corresponding values of $a$ determines the probability that the relationship apparently found was a result of chance coincidence. This probability is found to be $2.2 \times 10^{-9}$, which indicates that the relationship is a result of the chemical nature of the collagen.

TABLE 2.-Showing the whole-number relation among the experimental results

\begin{tabular}{|c|r|r|r|r|r|}
\hline$f$ & Weight & $\frac{f}{0.106}$ & $a$ & \multicolumn{1}{|c|}{$f / a$} & \multicolumn{1}{|c|}{$f / f_{0}$} \\
\hline 0.633 & & & & \\
\hline .397 & 2 & 5.97 & 24 & 0.02637 & 24.00 \\
.343 & 1 & 3.75 & 15 & .02647 & 15.05 \\
.317 & 1 & 2.99 & 13 & .02638 & 13.01 \\
.236 & 2 & 2.23 & 9 & .02642 & 12.02 \\
.184 & 1 & 1.74 & 7 & .02622 & 8.95 \\
.106 & 2 & 1.00 & 4 & .02650 & 6.98 \\
\hline
\end{tabular}

$f_{0}=0.02638$.

The reciprocal of $f_{o}$ is the number of nitrogen atoms occurring in a unit of the collagen structure, or a factor of this number. As the reciprocal of 0.02638 , the value found for $f_{o}$, is $37.91,38$ may be taken tentatively as the integer in question. The per cent of difference between $1 / f_{0}$ and 38 is slightly less than the probable percentage of error in the value of $f_{o}$, calculated from the distribution of the values f/a.

As there is a possibility that some integer other than 38 might fit the results better than 38 , a comparison was made of all integers less than 76 to determine the probability that they were not the correct numbers. The result for 38 was $1.1 \times 10^{-5}$ times that for 55 , the integer of this group having the next highest probability of being correct. The work was not carried further than for 75 because 38 gave a satisfactory correspondence with the experimental results.

According to Hofmeister's ${ }^{5}$ analysis, collagen contains 50.75 per cent carbon and 17.86 per cent nitrogen, which gives the atomic ratio of carbon to nitrogen as 3.318. Thus, if there are 38 nitrogen atoms in a unit of the collagen, there are (38) (3.318) atoms of carbon in the same unit. This product is 126.08 , which is sufficiently close to the integer 126 to provide a check on the determination of 38 as the number of nitrogen atoms.

Belden ${ }^{6}$ has measured the combination of gaseous $\mathrm{HCl}$ with gelatin. He found a combining weight of 332 for gelatin, which corresponds

S Z. physiol. Chem., vol. 2, p. 299, 1878.

J. Phys. Chem., vol. 35 , p. $2164,1931$. 
to a value of 0.237 for $f$. This checks the value of 0.236 obtained in the present work, for the fraction of the total nitrogen in collagen reacting under the same conditions. Belden also made this measurement on gelatin hydrolysate, the result being 285 to $290 \mathrm{mg}$ of $\mathrm{HCl}$ combined per gram of gelatin. This checks the value given in this paper for the air-dry gelatin which was kept in $\mathrm{HCl}$ at about atmospheric pressure for seven days, this value being $293 \mathrm{mg}$ of $\mathrm{HCl}$ per gram of gelatin.

The work of Thomas ${ }^{7}$ and Kelly must also be considered in connection with the results in this paper. The equivalent weights of collagen calculated from the results given in their papers are given in Table 3 , together with the corresponding values of $f$ and $a$. The values of $a / 38$ are given for comparison with the observed values of $f$. The fifth combining weight in the table is not given by Thomas and Kelly, but represents a maximum in the quantity of iron fixed by collagen in one of their experiments. The value of 94 from the work on chromium salts, reported by the authors, was derived from a similar maximum.

TABLE 3.-Combining weights of collagen with chromium and iron

[Data of Thomas and Kelly]

\begin{tabular}{|c|r|r|r|}
\hline $\begin{array}{c}\text { Combining } \\
\text { weight }\end{array}$ & $f$ & $a / 38$ & $a$ \\
\hline 94 & 0.836 & 0.842 & 32 \\
187 & .421 & .421 & 16 \\
380 & .207 & .211 & 8 \\
750 & .105 & .105 & 4 \\
299 & .263 & .263 & 10 \\
\hline
\end{tabular}

It should be noted that a necessary condition for the existence of the relation found among the experimental results is that the amount of physical adsorption taking place under the conditions of the experiments is negligible.

\section{CONCLUSIONS}

1. Collagen, as prepared for this work, is a chemical species.

2. The number of nitrogen atoms in a unit of the structure of collagen is 38 or a multiple of 38 . The corresponding number of carbon atoms is 126.

Washington, February 26, 1932.

7 See note 1, p. 549. 